\title{
An Ultra-Low Power Implantable Medical Devices: An Engineering Perspective
}

\author{
B. Sathyabhama ${ }^{1}$, B. Siva Shankari ${ }^{2}$ \\ ${ }^{1}$ Assistant Professor, Department of ECE, Panimalar Engineering College, Chennai. \\ ${ }^{2}$ Teaching fellow, Department of Information Science \& Technology, Anna University, Chennai.
}

\begin{abstract}
:
Implantable Medical Devices (IMDs) reside within human bodies either temporarily or permanently, for diagnostic, monitoring, or therapeutic purposes. IMDs have a history of outstanding success in the treatment of many diseases, including heart diseases, neurological disorders, and deafness etc., With the ever-increasing clinical need for implantable devices comes along with the continuous flow of technical challenges. Comparing with the commercial portable products, implantable devices share the same need to reduce size, weight and power. Thus, the need for device integration becomes very much imperative. There are many challenges faced when creating an implantable medical device. While this paper focuses on various techniques adapted to design a reliable device and also focus on the key electronic features of designing an ultra-low power implantable medical circuits for devices and systems.
\end{abstract}

Keywords: Implantable medical devices, low power, biocompatible materials, bio amplifiers, neural amplifiers, CMOS, FINFETs.

\section{Introduction}

An Implantable Medical Device (IMD) is either partly or totally introduced into the human body by surgically or medically and it is intended to remain there after the procedure. Implants deliver medication, monitor the body functions, or provide support to the organs and tissues. Implantable medical devices in some cases implanted for long term and contain electronic circuits and batteries either replaceable or rechargeable . Many of today's implantable devices use wireless technology to supply power and provide communication paving a path for better internet of medical things (IOMTs). The IOMTs refers to the connected system of medical devices and applications that collects the data that is then provided to healthcare IT systems through online computer networks for further diagnosis. Medical implants are generally made from skin, bone or other body tissues, plastic, ceramic or by several biocompatible materials (titanium and its alloys, aluminium, cobalt-alloys, stainless steel, poly-ethylene, polyurethanes, polyglycolide and polylactides) that have been commercially used for fabrication [1]. 


\section{WIRELESS IMPLANTABLE MEDICAL DEVICES}

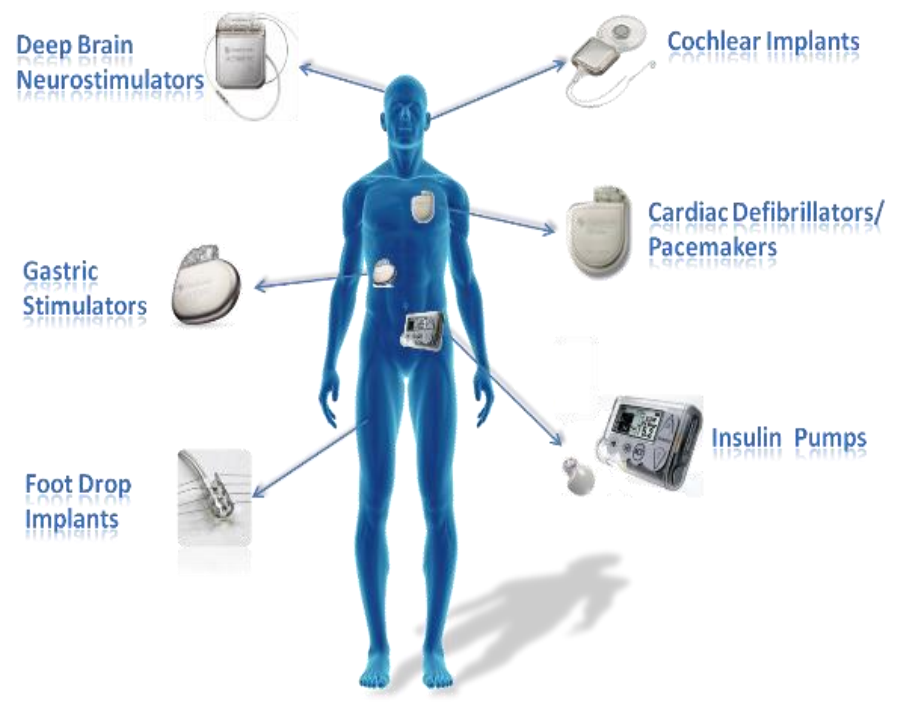

Figure 1: Examples of wireless implantable medical devices

These implants can be placed permanently or they can be removed once they are no longer needed. For example, cardio stents or hip implants are intended to be permanent in human body. But chemotherapy ports or screws to repair broken bones can be removed when they no longer needed [2]. The latest generation transistors evolved its pathway towards medical electronics on electrochemical biosensors such as fin field effect transistors (FinFETs), tunnelling field effect transistors (TFETs), and organic electrochemical transistors (OECTs) etc., Figure 1 shows the various examples of wireless implantable medical devices currently used.

\subsection{Design Challenges}

The first pacemaker was implanted in 1958, from that numerous engineering and medical activity development for IMDs have faced various challenges in materials, battery power, functionality, electrical power consumption, size shrinkage, system delivery, and wireless communication protocol and also issues such as reliability and fast bidirectional data communication, efficient power delivery to the implantable circuits, low noise and low power for the recording information for the system, and delivery of safe and correct stimulation to prevent tissue and electrode damage are some of the major challenges faced by the microelectronics circuit designer.

Due to the nature of physiological signals and the integrated circuits designed for medical applications should consume low power and to have low cutoff frequency, and low input-referred noise. The important features to be considered for designing the analog front-end circuits are subthreshold circuits, bulk-driven MOSFETs, floating gate MOSFETs, and log-domain circuits etc., to reduce power consumption. Some physiological signals, for example electrocardiogram (ECG), electroencephalogram (EEG), and electromyogram (EMG), chemical quantities such as ion concentrations and physical quantities such as body temperature, blood pressure, are often small signals of low frequency [3] as shown in figure 2. 


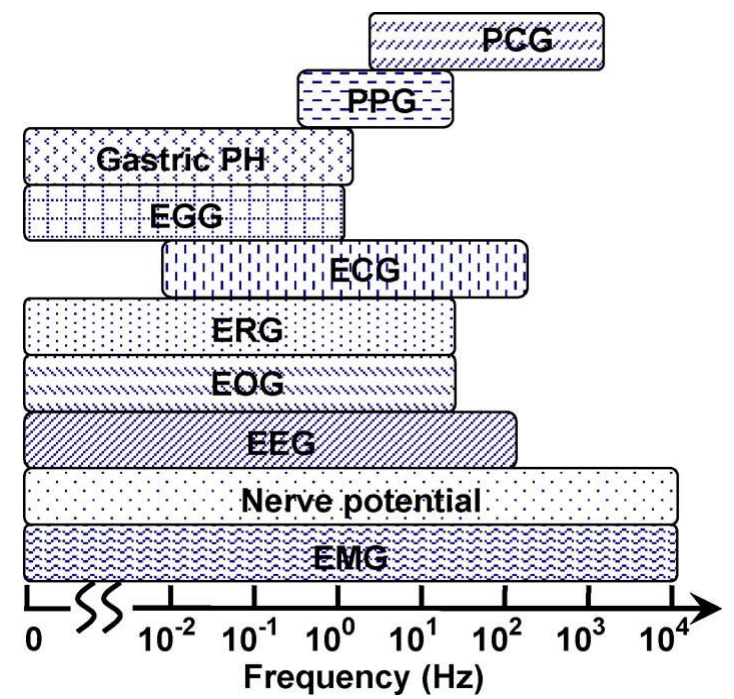

Figure 2. Frequency ranges of some physiological signals

where PCG, PPG, EGG, ECG, ERG, EOG, EEG, and EMG refer to the phonocardiographic, photoplethysmographic electrogastrographic, electrocardiographic, electroretinographic, electrooculographic, electroencephalographic and electromyographic signals respectively. After converting a physiological signal into an electrical signal by the corresponding type of sensor or transducer, an Analog Front End (AFE) circuit is often needed to filter and amplify the signal before digitizing it for further processing. The design of analog ICs for implantable medical devices faces three challenges mainly [3].

Firstly, the design must incorporate low power design techniques to reduce the amount of heat dissipation such that the surrounding human tissues will not get damaged or destroyed. Ultra-low power IC(Integrated Circuit) design is also important to be used along with new technologies related to battery to avoid the use of bulky batteries or frequent replacement of batteries during long-term operations.

Secondly, the nature of physiological signals are often low frequency signals that span from dc to a few kilohertz. Sometimes, there is also a large dc component caused by electrode and skin interface residing in the signal. Hence, the analog front-end integrated ciruits are often implemented with low cutoff frequencies to read out the signals from the electrodes or sensors. Such designs require large resistances and/or large capacitances, which can be easily achieved with discrete components but are difficult to be fabricated on chip directly due to the large areas they occupy.

Thirdly, the amplitude of many physiological signals can be relatively small and in the range of a few microvolts to tens of millivolts. Thus, the quality of the signals is largely affected by the noises from the electrodes or sensors, the power supplies, as well as by the user's motion. Therefore, to precisely process the weak physiological signals the circuits must exhibit low input referred noises. The major challenges of implantable are the minimum size and weight of chronic power supply, the package, and the transducers (sensor and the stimulator electrodes) integrated with signal processing electronics. 
Moreover, in emerging nanoscale technologies, the degraded circuit parameters (e.g. noise immunity), electrostatic discharge (ESD) properties, the maximum current densities, parasitic effects, process fluctuations, aging effects and other parameters are superior in bulk CMOS processes using planar transistors. So, this is the main reason, for using low voltage, low power high performance analog and mixed signal circuits for mature process nodes. With the technology development, the threshold voltage $(V T H)$ of MOS devices is not lowered with the same pace as the supply voltage scaling. Moreover, lesser value of supply voltage influences the main parameters of AFE circuits, such as dynamic range (DR), power supply rejection, noise immunity, etc. Figure 3. Shows the generic system architecture for implantable medical devices [4].

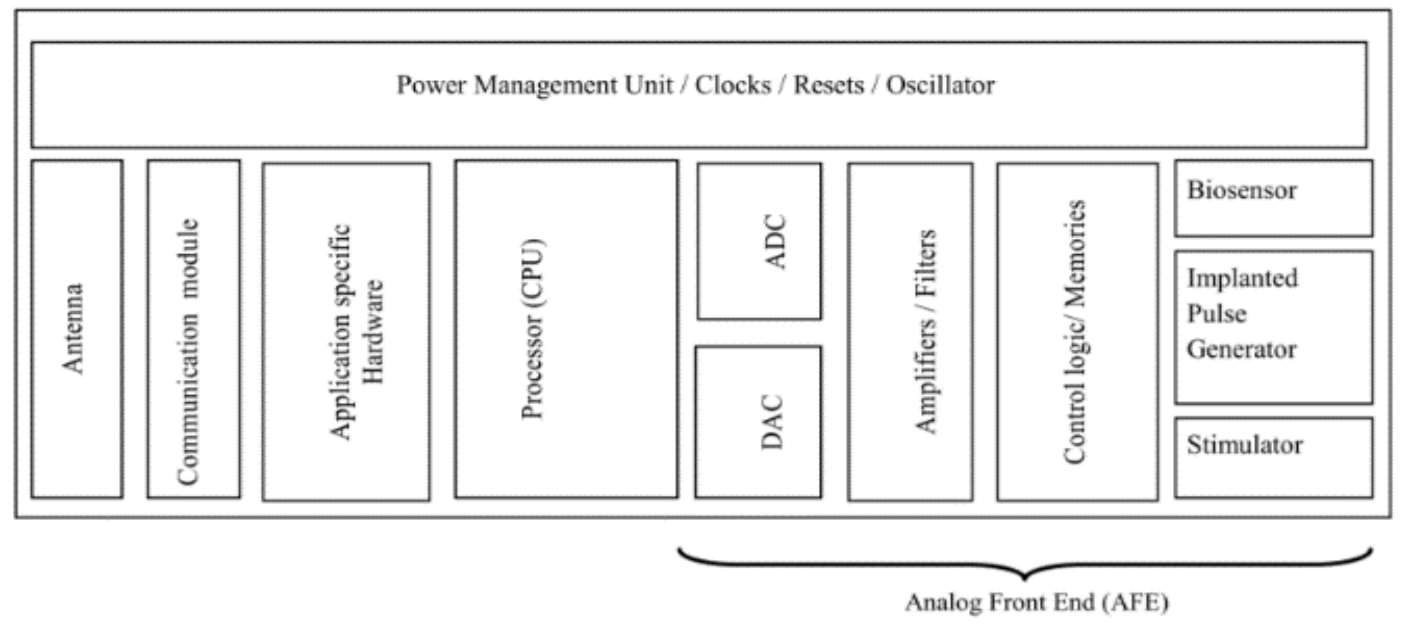

Figure 3. Generic system architecture for implantable medical devices.

\subsection{Security and privacy issues in IMDs:}

Evolving from a mere electromechanical IMD to one with more advanced computing and communication capabilities such as telemetry has many benefits but also entails numerous security and privacy risks for the patient [6]. A significant rule for pledging safety in the design of medical devices is that the device remains safe even under single fault conditions that could incite critical failures. An increasing number of IMDs are furnished with a wireless interface that allows monitoring and reprogramming non-invasively through an external device, also known as device programmer. This wireless interface also brings important security and privacy risks that may lead to remote attacks. The usage of wireless communication facilities in many modern IMDs are a major source of security risks, particularly while the patient is in non-medical environments where the implant becomes invisible, as its presence could only be remotely detected. Also, it eases the access to transmitted data by eavesdroppers who simply listen to the insecure channel. This may lead to a major privacy breach, as IMDs store sensitive information such as diagnosed conditions, vital signals, therapies, and a variety of personal data (e.g., date of birth, name, and other medically relevant identifiers).

Conserving the confidentiality of the transmitting information as well as the stored in information of the IMD is another mandatory aspect. The device must implement suitable security 
policies that limit what entities can reconfigure the IMD or get access to the information stored in it, ensuring that only authorized operations are executed. Similarly, the security mechanisms have to be implemented to shield the content of messages exchanged through an insecure wireless channel [6].

To provide security for any medical devices, adaptable are crucial, especially for the previously implanted devices. The authentication algorithms should prevent the access of unauthorized users to IMD, in case of an emergency, where the patient is not able to inactivate the authentication mechanism, IMD should be available to a doctor for an emergency treatment, even if the person is not authorized previously. Security mechanisms presents additional components to the system or run extra algorithms which in turn increases the susceptibility of IMDs against software bugs and hardware impairments, and lead to malfunctions. Hence, security mechanisms should be robust enough to ensure system reliability.

\subsection{Role of Wireless communication:}

For surveillance or monitoring of the device, extraction of the device or wired electrical connection to the device is necessary but preferably Wireless communication is used. Wireless communication transfers data or signals at some distance without the support of wire. The efficiency of data transfer will vary for each and every media. Mostly, the signal is very easily dispersed in a conductive media. If the frequency is higher, as much as a giga-hertz, the dissipation rate is also relatively higher. Therefore, the US Federal Communications Commission and European Telecommunications Standards Institute have defined the Medical Implants Communication Services band (402-405 MHz) (MICS) RF band) as the implantable medical device frequency range to support the connectivity standards and communication protocols which is characterised by capability of achieving 100's of $\mathrm{Kb} / \mathrm{sec}$ data transfer rates at a range of around $2 \mathrm{~m}$. This eliminates the need for external controller to be in a close vicinity as in case of inductive links. MICS provides the higher data rates, as required to upload patient procedures captured and stored in the IMD's memory to the base station for analysis and gives improved measurements and control in different IMD applications especially cardiac and neural applications [4]. The range limitations of inductive systems overcame by the MICS band and helps in the development of next generation medical devices with advanced patient health care.

\subsection{Designing low power electronics:}

\section{A. Analog Front End}

Analog front end IC of implantable medical devices consist of various blocks such as bio amplifiers, data converters, filters etc, Generally, biological/bioelectric signals have low amplitude and low frequency. Therefore, to increase the amplitude level of bio signals, amplifiers are designed. The designed amplifier outputs are used for further study and they are represented as ECG, EMG, or any bioelectric waveforms. Such amplifiers are defined as Bio Amplifiers or Biomedical Amplifiers [9]. 


\section{Basic Requirements for Biological Amplifiers}

1. The bio amplifier should have a high input impedance of the value ranging between $2 \mathrm{M} \Omega$ and $10 \mathrm{M} \Omega$ based on the applications. The distortion of the signal can be reduced by having a higher impedance value.

2. When electrodes pick up biopotentials from the human body, the input circuit should be protected so that, all bio-amplifiers should consist of isolation and protection circuits, to prevent the patients from electrical shocks.

3. The voltage gain value of the amplifier should be higher than $100 \mathrm{~dB}$ because the output of a bioelectric signal is always in millivolts or microvolt range.

4. A constant gain should be maintained throughout the entire bandwidth range.

5. A bio-amplifier should have a small output impedance and also it should be drift and noise free.

6. Common Mode Rejection Ratio (CMRR) value of amplifier should be greater than $80 \mathrm{~dB}$ to reduce the interference from common mode signal.

7. The gain of the bio-amplifier should be calibrated for each measurement [15].

Although different types of Bio Amplifiers (Differential Amplifier, Instrumentation Amplifier, Operational Amplifier, Neural amplifier, Isolation Amplifier, etc.,) are available, frequently used for the design of implantable medical devices are discussed here.

\section{Neural Amplifiers:}

Neural amplifiers are used to acquire untethered/portable neural recordings, as they meet the requirements of a miniaturized form factor, light weight and low power consumption. Furthermore, such miniaturized and low-power IC neural amplifiers are now being used in emerging implantable neural prosthesis technologies [16]. Generally neural amplifiers are designed with low input-referred noise and high mid-band gain. It is important to design the neural amplifiers with low power consumption and small silicon area. The input-referred noise of neural amplifiers for recording is typically kept below 5-30 $\mu \mathrm{V}$. When designing implantable microsystems to record tens to hundreds (or even thousands) of neural signals in parallel, neural amplifiers need to be small in physical dimensions and therefore expected to consume small chip area. The general block diagram representing the neural recording system is shown in figure 4 .

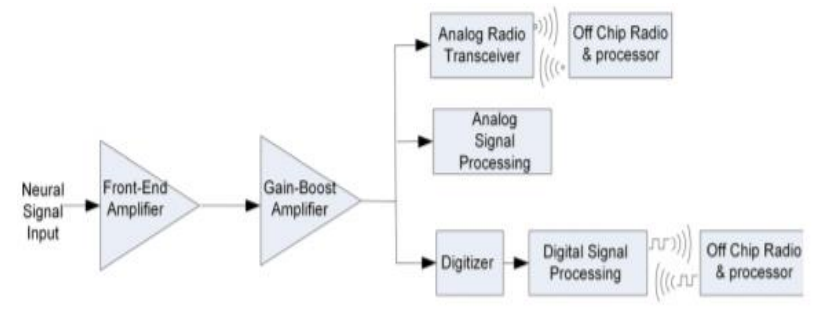

Figure 4. General system-on-chip block diagram of neural recording ICs.

The low power neural amplifier, is important from two standpoints. First, affordable power budget for the operation of implantable microsystems is limited, whether they are battery operated or 
telemetry powered. Secondly, from a biological point of view, the heat generated by the circuits that dissipate rather large power can potentially cause damage to the neighbouring living tissues. An amplifier's input referred noise with respect to its current consumption and bandwidth is called noise efficiency factor (NEF). The NEF is defined as:

$$
\mathrm{NEF}=V_{\mathrm{irms}} \sqrt{\frac{2 I_{\mathrm{tot}}}{\pi \cdot U_{t} \cdot 4 \kappa T \cdot \mathrm{BW}}}
$$

where $\mathrm{V}_{\text {irms }}$ is the input-referred root mean square (RMS) noise voltage, $\mathrm{I}_{\text {tot }}$ is the total amplifier bias current, $\mathrm{U}_{\mathrm{t}}$ is the thermal voltage, $\kappa$ is the Boltzmann's constant, $\mathrm{T}$ is the absolute temperature, and BW is the effective noise bandwidth. Neural amplifiers usually need to have high commonmode rejection ratio (CMRR) and a high power-supply rejection ratio (PSRR) in order to suppress supply voltage variations while amplifying the input neural signal by a rather large gain.

\section{Instrumentation Amplifiers:}

An input preamplifier is considered to be an important stage of all biopotential amplifiers which substantially contributes to the overall quality of the system. The main tasks of the preamplifier are to sense the voltage between two measuring electrodes while rejecting the common mode signal, and minimizing the effect of electrode polarization overpotentials [17]. In biomedical applications, high input impedance and high gain are attained with an instrumentation amplifier. Usually, a 3-stage amplifier setup forms the instrumentation amplifier circuit. The transducer output is given as input to the instrumentation amplifier. A special amplifier is required with high CMRR, high input impedance and to avoid loading effects, before the signal goes to the next stage. The non-inverting amplifier is connected to each input of the differential amplifier. As shown in figure 5 the amplifier on the left side acts as non-inverting amplifiers and they are combined together to form the input stage of the instrumentation amplifier. The third stage operational amplifier is the difference amplifier, and it gives the output of the instrumentation amplifier.

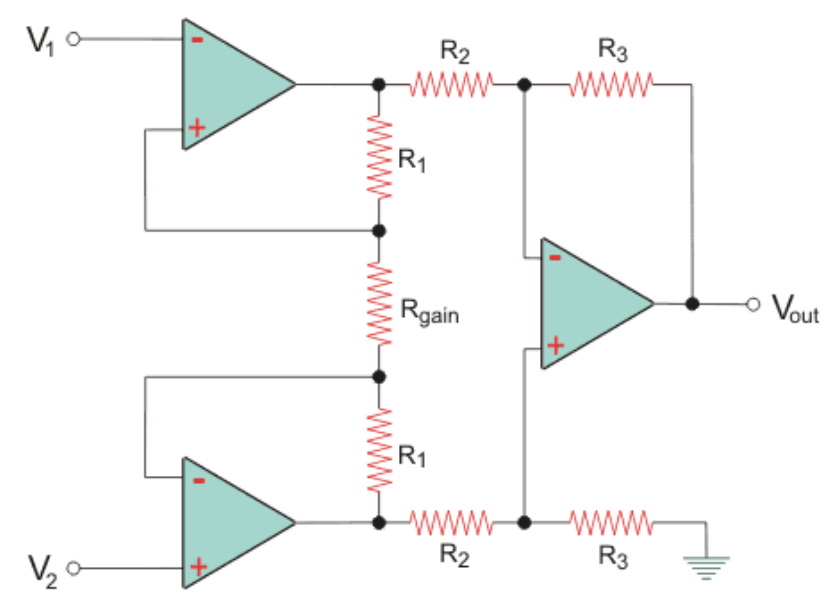

Figure 5: Instrumentation Amplifier 
The output from the difference amplifier $\mathrm{V}_{\text {out }}$ is the difference between two input signals given at the input points.

$$
V_{\text {out }}=\frac{R_{3}}{R_{2}}\left(V_{O 1}-V_{O 2}\right)
$$

$\mathrm{V}_{\mathrm{O} 1}$ is the output from op-amp 1 and $\mathrm{V}_{\mathrm{O} 2}$ is the output from op-amp 2.

\section{Isolation Amplifiers:}

Isolation amplifiers are known as Pre-amplifier isolation circuits. The main purpose of the isolation amplifier in a biopotential amplifier, is the protection of the patient by eliminating the hazard of electric shock resulting from the interaction among patient, amplifier, and other electric devices in the patient's environment, specifically defibrillators and electrosurgical equipment [17]. Isolation amplifiers can be used to eliminate source ground connections, break ground loops, and provide isolation protection to patient and electronic equipment. It also prevents the line frequency interferences and accidental internal cardiac shock. It provides up to $1012 \Omega$ insulation between the patient and the power line in the hospital [10]. The block diagram of isolation amplifier is shown

in

(a)

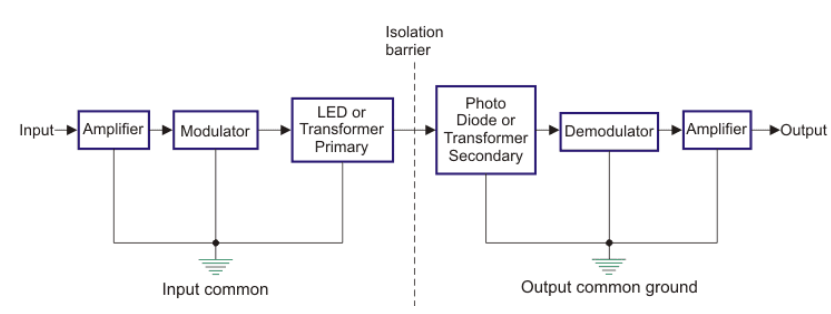

(b)

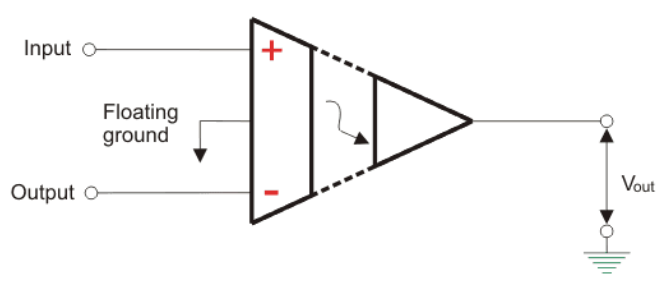

Figure 6. (a) Block diagram of isolation amplifier (b) Symbol of isolation amplifier.

The electrical signals are obtained with electrodes. The received signals goes to the amplifier block, where signals amplification occurs. After amplification, the signal pass in the modulation block. When it goes to the isolation barrier, transformer or optical cable can be used. If the transformer acts an isolation barrier, the modulator output connects to the primary winding of the transformer. Energy from primary winding transfers to the secondary winding based on the mutual induction principle. But in case of optical cable, modulator output goes to LED. Where the electrical signals gets converted into light energy. At the later stage, the secondary output enters the demodulation block. Finally, the amplified demodulated signal is obtained as an output.

\section{B. Power Management Circuit}

One of the most tough challenges for implanted medical device is to provide sufficient power to record and transmit signals. However, there has been great progress in understanding how to design miniature low power circuits for biological applications [5]. With the progress in ultra-low power design, the concept of energy harvesting to ultra-low power (ULP) electronic circuits has 
also gained relevance [18]. The most common method used for powering larger medical implants such as pacemakers and deep brain stimulation devices is via batteries.

However, miniaturizing the size of the batteries are very difficult and remain the size-limiting component for many medical implants. In addition, the lifetime of batteries limits the longevity of the potential implants. Replacing the battery for implantable medical devices often requires an additional surgery and can cause many complications to the patient. Alternatively, rechargeable batteries pave a way for longer lifetimes but need an additional circuitry for delivering power to recharge, such as RF approaches, which suffer from low-efficiency power transfer and require relatively large, aligned antennas. The amount of power wirelessly transferred is limited and any excessive power drained by the medical implant can compromise the operation of the device and cause tissue heating in the surrounding medium. Since multi-channel arrays are used, each individual channel should be designed to consume as little power as possible to manage with the overall reduction in power consumption for the implanted medical devices. Furthermore, smaller die area in the interface IC is also preferable with increased number of arrays for higher spatial resolution which in turn provides a good relevance [7].

\section{Energy harvesting techniques:}

The concept of energy harvesting from human body motion and environmental sources for implantable devices has gained a new relevance. The implants that are powered by harvested energy are comparatively more convenient and useful to the patients. The form of energy that is used to scavenge the power using the harvesting techniques defines the type of energy harvesting [18]. Figure 7 shows the different types of energy harvesting methods in implanted medical devices.

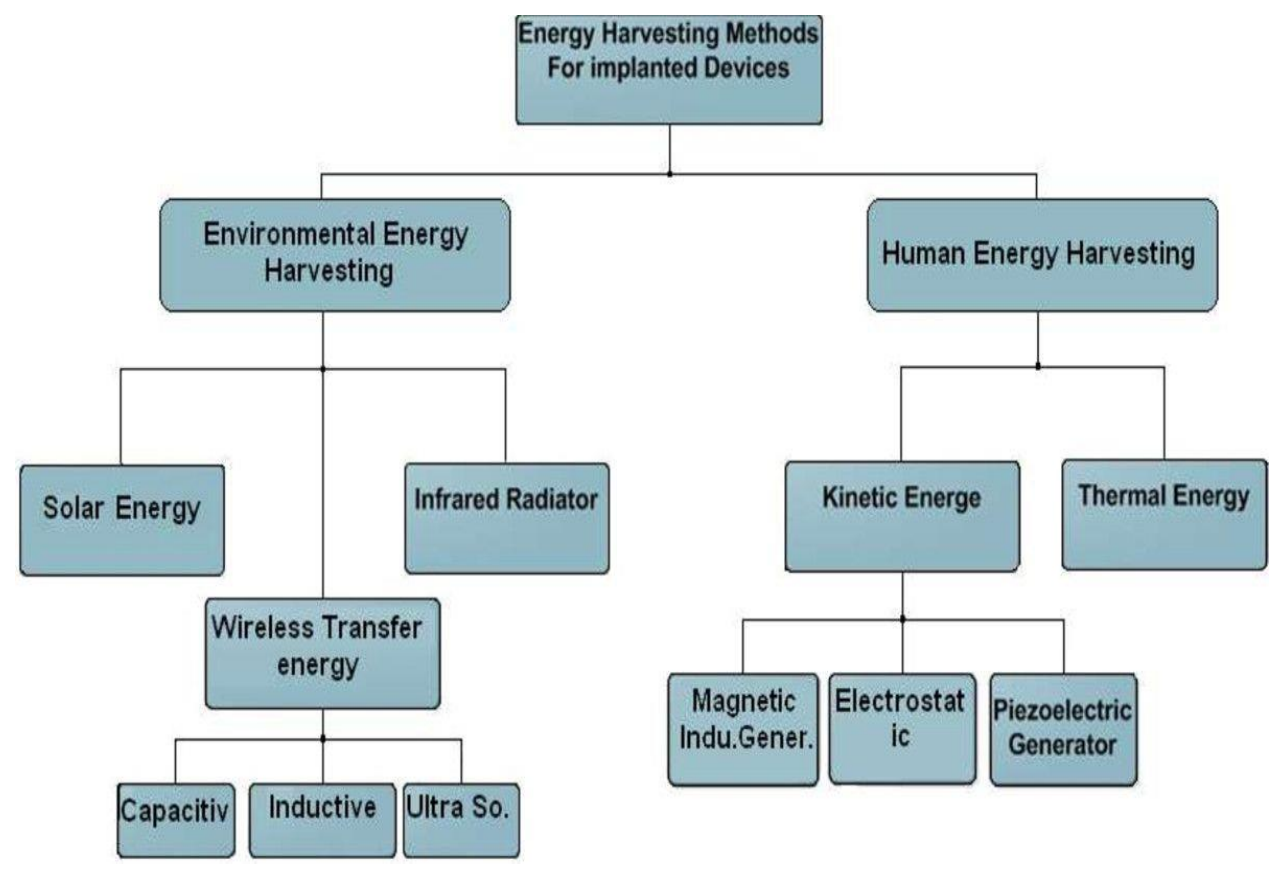

Figure 7. Types of energy harvesting in implanted medical devices. 
However, sufficient amount of kinetic energy and chemical energy is produced in the human body itself. Even if a small fraction of the biomechanical energy released during cardiac contraction and relaxation could be harvested and used to power a cardiac pacemaker, then it may represent a promising alternative to solve the problems of limited battery life of Implantable Medical Electronics (IMEs) [8].

Biochemical energy harvesters that use the glucose in human body as a fuel exhibit the potential of energy utilization from the body of living things. This can be used in different biological applications including local glucose concentration monitoring for diabetes management, local temperature monitoring for infection control after surgery, blood pressure measurement to indicate blockage of fluid flow in the central nervous system etc.

Pyroelectric energy harvesters usually generate output power from the temperature gradient, but the output is not sufficient enough to be useful in many practical situations [8].

\section{Communication Protocol}

Communication with implanted medical devices is usually provided with a wired connection or with wireless radiofrequency (RF) telemetry. However, wires can break, become damaged or introduce noise in the recording through movement artifacts or by antenna effects. Communication with wired connection is frequently reported with complications in deep brain stimulation devices and with pacemakers and implantable cardioverter defibrillators (ICDs). Medical Implants Communication Services band (402-405 MHz) is used as implantable medical device frequency range [11] for communication purpose. Due to the safety-critical nature of IMDs, an implant requires a robust security protocol that does not allow access to individuals without knowledge of a secret password. At the same time, the protocol has to be accessible enough to allow unrestricted access to any first-aid responder with medical expertise in case of patient (e.g., heart attack, seizure) or device (e.g., malfunction, low battery) emergency [20].

\section{IMDs with telemetry:}

Healthcare systems with numerous networking functions and communication have proliferated over the last years. This has made possible to develop medical sensor networks, for example, patients can be monitored from their homes itself. Hence, wireless telemetry has become a close part of high performance implantable biomedical devices. The designed wireless interface system should be capable of both receiving data from the implant, and sending power and data to the medical implant. Therefore, the wireless interface is typically consisted of a voltage regulator (power telemetry), rectifier and data receiver (forward telemetry), and data transmitter (reverse telemetry). The IMD can communicate with an external device generally known as "Programmer" or "Reader'" and send it physiological data such as electrocardiogram (ECG) signals in the case of pacemakers and ICDs, so that doctor can use it to trail the patient's pathology. Apart from querying the sensed data, the Programmer can also command the IMD to adjust or disable therapies, perform software updates, etc [6]. 
Wireless RF telemetry has been used in several implantable medical devices to avoid the complications of wired medical implants. However, wireless Radio Frequency (RF) telemetry requires substantial power and suffers from poor transmission through biological tissue. RF telemetry also is in need of a relatively large antenna, which limits the size of the implantable devices and prevents implantation in organs such as heart, brain, and spinal cord without causing significant damage to the neighbouring tissue. Other methods of wireless communication have been investigated to communicate with implants, including optical [21] and ultrasound [22]. However, these methods also have low-efficiency transmission through the body and would be difficult to miniaturize when trading off towards size.

Intrabody communication is a recently developed alternative method of wireless communication, which uses the conductive properties of the body to transmit signals [19].

\subsection{Emerging designs in IMDs:}

The latest generation transistors, electrochemical biosensors (fin field-effect transistors (FinFETs), tunnelling field-effect transistors (TFETs), and organic electrochemical transistors (OECTs)), and the combination of electrochemical biosensors with lab-on-chips are greatly used for medical applications [12]. The constant scaling of planar metal-oxide-semiconductor fieldeffect transistor (MOSFET) has technological difficulties whereby the device can no longer be classified as a long channel MOSFET. The main short-channel effect is due to two-dimensional distribution of potential and high electric fields in the channel region, which mainly lead to difference in the threshold voltage, saturation region that does not depend on drain current and drain potential. Subsequently a variety of different non-planar and planar topologies were investigated, such as the FinFET and the TFET. The FinFET resulted in best option for the fabrication of a non-planar device with self-aligned double-gate using a standard complementary metal-oxide semiconductor (CMOS) process. Also, the main reason of using FinFET is low power operation, lower voltage of operation, higher speed and lower static power consumption in the device.

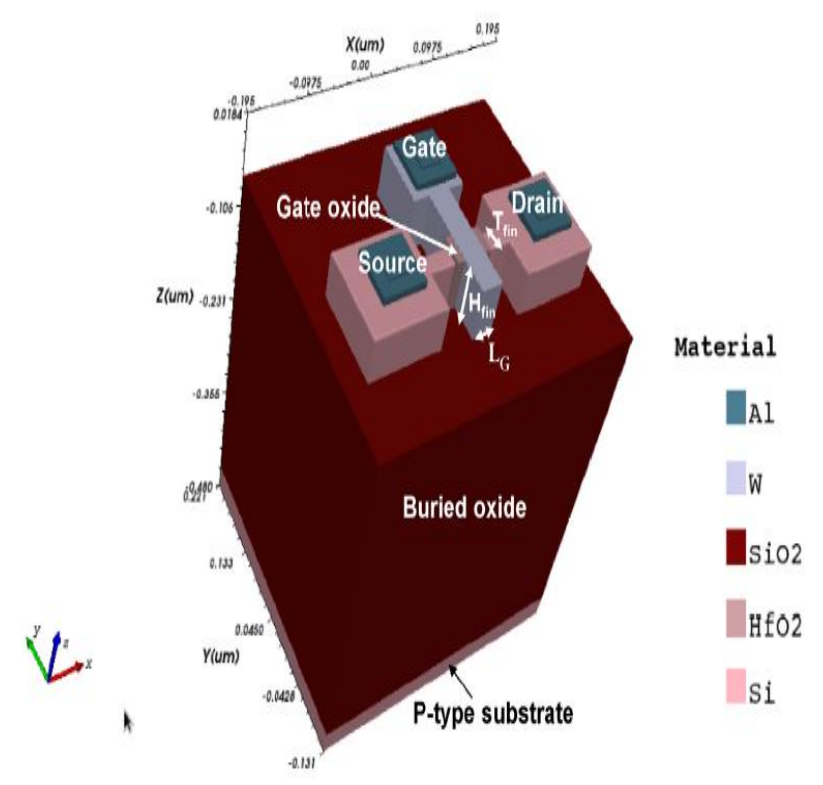


The design of SOI FinFET device-based Instrumentation Amplifier (IN-Amp) for biomedical applications. An IN-Amp is operated in weak inversion region effectively for low power and low voltage applications. The performance metrics of the proposed IN-Amp such as Common mode rejection ratio (CMRR), DC gain, were enhanced by using nanometre FinFET device technology. To achieve these required performance metrics, size of transistors is optimized and passive resistances are replaced by active $30 \mathrm{~nm}$ SOI FinFET transistor. A FinFET based DC offset removal circuit is considered to reduce the offset voltage at the output of the IN- amp. The output achieved by affixing DC offset removal circuit is exact replica of input over wide range of frequency, which can be adjusted by varying clock inputs to the circuit [10].

In Biomedical applications the nature of the biomedical connection is resolved in huge part by the qualities of the VCO (Voltage Controlled Oscillator). Generally, VCOs utilizing CMOS innovation have been utilized for low recurrence applications, however $18 \mathrm{~nm}$ forms in FINFET technology have permitted CMOS oscillators to accomplish frequency in the gigahertz extend. This range is made conceivable with the utilization of programmed swing control [13].

For emerging designs using the lower technology nodes, the adiabatic or energy recovery logic design approach, a non-conventional low power design methodology is identified to be an alternative circuit design approach for conventional CMOS circuit designs. The adiabatic logic retrieves a significant amount of energy from the output nodal capacitances to the power clock source every time the output node switches to logic 0 . The recovered energy is reused in the operation of adiabatic logic circuit [23].

\section{Conclusion:}

In this paper, considerations in the development of implantable medical devices were reviewed from an engineering viewpoint. Engineers pour their technical knowledge into the practical design of the devices. Biocompatibility and durability of the material exposed to the inner body environment must be guaranteed by a series of laboratory experiments, animal studies, and so on. Many simulations, prototypes, and testing help to obtain the best device. The electrical power source is carefully designed to get a suitable shape and the power supply is continuously monitored to ensure uninterrupted operation. For real-time monitoring of IMDs in the human body, active or passive wireless communication methods are selected with contestation of packaging material, power, and detection interval. Developing medical devices for the human body must be initiated by an understanding of the environment, that is, a human being.

\section{References:}

1. Suman Chatterjee, Mansi Saxena, Deepak Padmanabhan, Mahesh Jayachandra, Hardik J.Pandya, "Futuristic medical implants using bioresorbable materials and devices", Elsevier- Biosensors and Bioelectronics, volume 142, 1 October 2019, 111489. 
2. B.Behrens, A.Bouguecha, M.Lerch, H.Windhagen, A.Almohallami, "Influence of Hip Prosthesis Size and Its Coating Area on Bone Remodeling", IEEE Transactions on NanoBioscience, Volume: 16, Issue: 8, Dec. 2017.

3. Yan Li, Carmen C. Y. Poon and Yuan-Ting Zhang, "Analog Integrated Circuits Design for Processing Physiological Signals", IEEE reviews in biomedical engineering, vol. 3, 2010.

4. Vipan Kakkar, "An Ultra Low Power System Architecture for Implantable Medical Devices” IEEE Access August 2019.

5. Sarpeshkar R, "Ultra Low Power Bioelectronics: Fundamentals, Biomedical Applications, and Bio-Inspired Systems. Cambridge University Press; Cambridge, UK: 2010.

6. Carmen Camara, Pedro Peris-Lopez, Juan E. Tapiador, "Security and privacy issues in implantable medical devices: A comprehensive survey", Journal of Biomedical Informatics, Elsevier, 2015.

7. Alfian Abdi, Hyouk-Kyu Cha, "A bidirectional neural interface CMOS analog front-end IC with embedded isolation switch for implantable devices”, Microelectronics Journal, Elsevier, 2016.

8. Asif Abdullah Khan, Alam Mahmud, and Dayan Ban, "Evolution From Single to Hybrid Nanogenerator: A Contemporary Review on Multimode Energy Harvesting for SelfPowered Electronics”, IEEE Transactions On Nanotechnology, Volume 18, 2019.

9. Fatemeh Karami Horestani, Mohammad Eshghi, Mohammadreza Yazdch, "An ultra-low power amplifier for wearable and implantable electronic Devices", Microelectronic Engineering, ELSEVIER 2019.

10. Reena Sonkusare, Omkar Joshi, S.S. Rathod, "SOI FinFET based instrumentation amplifier for biomedical applications”, Microelectronic Engineering, ELSEVIER 2019.

11. Yeun-Ho Joung, "Development of Implantable Medical Devices: From an Engineering Perspective", International Neurourology Journal, 2013.

12. Maria Laura Coluccio, Salvatore A. Pullano, Marco Flavio Michele Vismara et al, "Emerging Designs of Electronic Devices in Biomedicine" Micromachines, 2020.

13. M. Santhosh Rani A, K. Vinothkumar, "Design of low power VCO using FinFET technology for biomedical applications” Materials Today: Proceedings, ELSEVIER 2020.

14. Andreas Demosthenous, "Advances in Microelectronics for Implantable Medical Devices" Advances in Electronics, Hindawi Publishing Corporation, 2014.

15. R.S Khandpur, "Handbook of bio medical instrumentation" Mcgraw hill publication, $3^{\text {rd }}$ edition, 2014.

16. Kian Ann Ng, Elliot Greenwald, Yong Ping Xu, Nitish V. Thakor, "Implantable neurotechnologies: a review of integrated circuit neural amplifiers", Medical \& Biological Engineering \& Computing, 2016. 
17. Nagel, J. H. "Biopotential Amplifiers", “The Biomedical Engineering Handbook”, Second Edition, CRC Press LLC, 2000.

18. Sakshi Koul, Suhaib Ahmed, Vipan Kakkar, "A Comparative Analysis of Different Vibration Based Energy Harvesting Techniques for Implantables", International Conference on Computing, Communication and Automation, IEEE, 2015.

19. John E Ferguson and A David Redish, "Wireless communication with implanted medical devices using the conductive properties of the body", Expert Review of Medical Devices, 2011.

20. Christos Strydis, Robert M. Seepers, Erasmus Mc Pedro Peris-Lopez, Dimitrios Siskos and Ioannis Sourdis, "A System Architecture, Processor, and Communication Protocol for Secure Implants", ACM Transactions on Architecture and Code Optimization, 2013.

21. Murakawa K, Kobayashi M, Nakamura O, Kawata S., "A wireless near-infrared energy system for medical implants”, IEEE Eng Med Biol. 1999.

22. Echt DS, Cowan MW, Riley RE, Brisken AF, “Feasibility and safety of a novel technology for pacing without leads", Heart rhythm, 2006.

23. B.P. Bhuvana, V.S. Kanchana Bhaaskaran, "Design of FinFET-based Energy Efficient Pass-Transistor Adiabatic Logic for ultra-low power applications", Microelectronics Journal, Elseveir, 2019. 